sehr kleines Volumen eingedampft war, krystallisierte das Bariumsalz der Tetramethyl-zuckersäure in langen Nadeln aus. Die Substanz wurde im Vakuum bei $100^{\circ}$ über Phosphorpentoxyd getrocknet. Sehr leicht löslich in Wasser.

$0,00789 \mathrm{gr}$ Subst. gaben $0,00857 \mathrm{gr} \mathrm{CO}_{2}$ und $0,00252 \mathrm{gr}_{2} \mathrm{O}$

$0,1928 \mathrm{gr}$ Subst. gaben $0,1118 \mathrm{gr} \mathrm{BaSO}_{4}$

$$
\begin{array}{llll}
\mathrm{C}_{10} \mathrm{H}_{16} \mathrm{O}_{8} \mathrm{Ba} & \text { Ber. C 29,90 } & \text { H 3,98 } & \text { Ba 34,24\% } \\
& \text { Gef. , 29,64 } & \text {, 3,57 } & \text {, 34,13\% }
\end{array}
$$

\title{
Tetramethyl-zuckersaures Silber:
}

Kochend heisse, wässerige Lösungen äquivalenter Teile tetramethylzuckersauren Bariums und Silbersulfats wurden unter Umrühren zusammengegeben. Vom abgeschiedenen Bariumsulfat wurde abfiltriert und das Filtrat im Vakuum zur Trockene gebracht. (Lichtabschluss.) Weisses Pulver, in Wasser sehr leicht löslich.

Zürich, Chemisches Laboratorium der Universität.

\section{Höhere Terpenverbindungen $\mathbf{V}^{1}$ ). Überführung der Abietinsäure ins Methylreten von}

\section{Ruzieka und Jules Meyer.}

(29. V. 22.)

Auf Grund der Überführung der Abietinsäure $\mathrm{C}_{20} \mathrm{H}_{30} \mathrm{O}_{2}$ durch Erhitzen mit Schwefel ins Reten $\mathrm{C}_{18} \mathrm{H}_{18}{ }^{2}$ ) (1-Methyl-7-isopropylphenanthren) ist die Stellung von $18 \mathrm{Kohlenstoffatomen} \mathrm{in} \mathrm{der} \mathrm{Molekel}$ dieser Säure festgelegt. 'Da wir kürzlich ${ }^{3}$ ) in der Abietinsäure die Anwesenheit von drei Ringen sichergestellt hatten, so kann man annehmen, dass die Abietinsäure ein hydriertes Phenanthrenderivat darstellt

1) IV. Mitt. Helv. 5, 369 (1922).

2) Vergl. die Literaturangaben Helv. 5, 316, Anm. 1-5 (1922).

3) Helv. 5, 315 (1922). 
und als Methyl-dekahydroreten-carbonsäure bezeichnet werden kann. Es müsste danach bei der Dehydrierung der Abietinsäure ausser der Carboxylgruppe noch ein weiteres Kohlenstoffatom abgespalten werden. Da bei dieser Operation neben Reten weder ein niederes noch ein höheres Homologes desselben beobachtet werden konnte, so liegt die Annahme nahe, dass die Eliminierung dieses einen Kohlenstoffatoms in seiner Bindungsart begründet sein muss und nicht etwa zufällig ist.

Eine analoge Beobachtung machten wir bei der Dehydrierung einiger Sesquiterpene $\mathrm{C}_{15} \mathrm{H}_{24}$ mit Schwefel zum Naphtalinkohlenwasserstoff Eudalin $\mathrm{C}_{\mathbf{1 4}} \mathrm{H}_{\mathbf{1 6}}$, wo dieses auch als einziges Dehydrierungsprodukt isoliert wurde. Im Gegensatz dazu lieferten uns andere Sesquiterpene das Cadalin $\mathrm{C}_{15} \mathrm{H}_{18}$ als alleiniges Dehydrierungsprodukt, also ohne Abspaltung von Kohlenstoff analog dem Übergang der Terpene in $\mathrm{p}^{-\mathrm{Cymol}^{1}}{ }^{1}$. Alle bisher untersuchten Dehydrierungen von Terpenverbindungen mit Schwefel verlaufen also eindeutig.

Es liegt in der Natur des Dehydrierungsvorganges begründet, wenn man die Vorstellung über die Bindungsart des abgespaltenen Kohlenstoffs dahin vervollständigt, dass demselben im hydrierten System eine Stellung zukommen muss, die im aromatischen Zustande nicht mehr möglich ist. Solche Stellungen sind die semicyclisch gebundene Methylengruppe (a), sowie die an einem Ringkohlenstoff,
a) $\underset{\mathrm{C}}{\mathrm{C}} \mathrm{C}=\mathrm{CH}_{2}$
b) $\stackrel{\mathrm{C}}{\mathrm{C}}>\mathrm{C}-\mathrm{CH}_{3}$

der mit drei weiteren Kohlenstoffatomen verknüpft ist, sitzende Methylgruppe (b). Da über die Lagerung der Doppelbindungen der Abietinsäure nichts sicheres bekannt ist, so untersuchten wir zunächst, um zwischen den obigen beiden Möglichkeiten entscheiden zu können, die Dehydrierung der Dihydro-abietinsäure, bei der eine Methylengruppe nicht mehr vorhanden sein kann. Bei dieser Operation entstand nur Reten, woraus zu folgern ist, dass das beim Erhitzen mit Schwefel abgespaltene Kohlenstoffatom der Abietinsäure einer Methylgruppe der oben unter b) näher charakterisierten Bindungsart entspricht. Die Dehydrierung der Abietinsäure (I) und der Dihydrosäure (II) kann demnach folgendermassen formuliert werden:
I. $\mathrm{C}_{18} \mathrm{H}_{26}\left\{\begin{array}{l}\mathrm{CH}_{3} \\ \mathrm{COOH}\end{array}+5 \mathrm{~S} \longrightarrow \mathrm{C}_{18} \mathrm{H}_{18}+\mathrm{CO}_{2}+\mathrm{CH}_{3} \mathrm{SH}+4 \mathrm{H}_{2} \mathrm{~S}\right.$
II. $\mathrm{C}_{18} \mathrm{H}_{28}\left\{\begin{array}{l}\mathrm{CH}_{3} \\ \mathrm{COOH}\end{array}+6 \mathrm{~S} \longrightarrow \mathrm{C}_{18} \mathrm{H}_{18}+\mathrm{CO}_{2}+\mathrm{CH}_{3} \mathrm{SH}+5 \mathrm{H}_{8} \mathrm{~S}\right.$

1) Vergl. darüber Helv. 5, 347-349 (1922). 
Wir sind dann dazu übergegangen, die Bindungsweise der Carboxylgruppe mit Hilfe der Dehydrierung näher zu untersuchen. Aus der Abspaltung der Carboxylgruppe beim Erhitzen der Abietinsäure mit Schwefel kann kein bestimmter Schluss gezogen werden, da wohl im allgemeinen aromatische Carbonsäuren bei diesen Bedingungen zur Kohlendioxydabspaltung neigen dürften. Man besitzt, darüber allerdings keine weiteren Erfahrungen. Wir dehydrierten daher den Methylester der Abietinsäure (III), bei dem eine direkte Kohlendioxydabspaltung weniger in Betracht kommt, aber auch hier war Reten das alleinige fassbare Dehydrierungsprodukt:

III. $\mathrm{C}_{18} \mathrm{H}_{26}\left\{\begin{array}{l}\mathrm{CH}_{3} \\ \mathrm{COOCH}_{3}\end{array}+5 \mathrm{~s} \longrightarrow \mathrm{C}_{18} \mathrm{H}_{18}+\mathrm{CO}_{2}+2 \mathrm{CH}_{3} \mathrm{SH}+3 \mathrm{H}_{2} \mathrm{~S}\right.$

Das gleiche war der Fall beim Erhitzen des Abietinols (IV), das wir durch Bouveault'sche Reduktion des Abietinsäure-methylesters herstellten, mit Schwefel:

IV. $\mathrm{C}_{18} \mathrm{H}_{26}\left\{\begin{array}{l}\mathrm{CH}_{3} \\ \mathrm{CH}_{2} \mathrm{OH}\end{array}+5 \mathrm{~S} \longrightarrow \mathrm{C}_{18} \mathrm{H}_{18}+\mathrm{CH}_{2} \mathrm{O}(?)+\mathrm{CH}_{3}{ }^{\prime} \mathrm{SH}+4 \cdot \mathrm{H}_{2} \mathrm{~S}\right.$

Die Deutung der Abspaltung sowohl der Carbonestergruppe wie auch der primären Alkoholgruppe könnte einmal darin gesucht werden, dass diesen beiden Substituenten - und somit auch dem Carboxyl in der Abietinsäure eine Stellung zukommt, aus der sie beim Übergang in das aromatische Ringsystem strukturmässig entfernt werden müssen. Es ist aber ferner möglich, dass durch Schwefel allgemein derartige Substituenten ohne Rücksicht auf die Bindungsart eliminiert werden. Auch in dieser Beziehung ist der Mechanismus des Dehydrierungsvorganges noch nicht näher erforscht.

Es schien uns daher nötig zu sein, die Dehydrierung an einem sauerstoffreien Derivate der Abietinsäure vorzunehmen. Beim Behandeln des Abietinols mit Phosphorpentachlorid wird hauptsächlich Wasser abgespalten unter Bildung eines Kohlenwasserstoffs $\mathrm{C}_{\mathbf{2 0}} \mathrm{H}_{\mathbf{3 0}}$, den man als Methylabietin ${ }^{1}$ ) bezeichnen kann und für den die Formeln

$$
\mathrm{C}_{18} \mathrm{H}_{25}\left\{\begin{array} { l } 
{ - \mathrm { CH } _ { 3 } } \\
{ = \mathrm { CH } _ { 2 } }
\end{array} \quad \mathrm { C } _ { 1 8 } \mathrm { H } _ { 2 4 } \left\{\begin{array}{l}
-\mathrm{CH}_{3} \\
-\mathrm{CH}_{3}
\end{array}\right.\right.
$$

in Betracht kommen, von denen die letztere infolge der Neigung der semicyclischen Doppelbindung zur Wanderung (bei den Versuchsbedingungen) in den Ring vorzuziehen ist. Das neben dem Methylabietin bei der Chlorierung wohl entstehende Chlorid des Abietinols, das nicht

1) Entsprechend der Bezeichnung des $\mathrm{C}_{19} \mathrm{H}_{28}$ als Abietin. 


\section{$-584$}

rein isoliert wurde, geht durch Chlorwasserstotfabspaltung mittels Chinolin gleichfalls ins Methylabietin über. Bei der Dehydrierung des letzteren (V) mit Schwefel entsteht nun ein neuer Phenanthrenkohlen-

$$
\text { V. } \mathrm{C}_{18} \mathrm{H}_{24}\left\{\mathrm{CH}_{3}+4 \mathrm{~S} \longrightarrow \mathrm{C}_{18} \mathrm{H}_{17} \cdot \mathrm{CH}_{3}+\mathrm{CH}_{3} \mathrm{SH}+3 \mathrm{H}_{2} \mathrm{~S}\right.
$$

wasserstoff $\mathrm{C}_{19} \mathrm{H}_{20}$, der seiner Bildungsweise nach ein Methylreten darstellen muss und dessen neue Methylgruppe die Stellung der Carboxylgruppe bei der Abietinsäure anzeigt. Auch bei dieser Operation konnte ausser Methylreten kein anderes charakterisierbares Dehydrierungsprodukt gefasst werden.

Da also die an Stelle des Carboxyls in die Abietinsäure eingeführte Methylgruppe beim Erhitzen mit Schwefel nicht abgespalten wird, so folgt daraus, dass die sauerstoffhaltigen Substituenten bei der Dehydrierung nicht infolge ihrer besonderen. Stellung, sondern auf Grund der Natur der Reaktion entfernt werden. Weiter ist damit eindeutig festgestellt worden, dass die Carboxylgruppe der Abietinsäure nicht tertiär ${ }^{1}$ ) gebunden sein kann, wie schon wiederholt von verschiedenen Forschern ${ }^{2}$ ) angenommen wurde. Da nach der schweren Esterifizierbarkeit der Abietinsäure auch die primäre Lage der Carboxylgruppe ausgeschlossen ist, kommt nur die sekundäre Bindungsart oder die an einer Ringdoppelbindung in Betracht. In letzterem Falle müsste die Abietinsäure eine $\alpha, \beta$-ungesättigte Säure sein. Da jedoch bei der Bouveault'schen Reduktion $\alpha, \beta$-ungesättigter Säure-ester ${ }^{3}$ ) auch die Kohlenstoffdoppelbindung hydriert wird, was aber beim Übergang des Abietinsäure-esters ins Abietinol nicht der Fall ist ${ }^{4}$ ), so muss die Abietinsäure eine sekundäre' Carbonsäure sein, und zwar auf Grund des Baus der ganzen Molekel eine mit direkt am Ring sitzender Carboxylgruppe.

Die bekannten Abbauversuche bei der Abietinsäure zeigen, in Analogie mit den Verhältnissen bei den Sesquiterpenverbindungen ${ }^{5}$ ), dass die Konstitutionsaufklärung allein durch direkten Abbau mindestens sehr schwierig sein wird.

Wir halten es daher auch hier, wie bei den Sesquiterpenen, für wünschenswert, die Stellung der Substituenten im Ringsystem wo-

1) D. h. an einem Kohlenstoffatom, das mit drei anderen C-Atomen verknüpft ist.

2) Vergl. z. B. P. Levy, Z, an. Ch. 81, 145ff. (1913).

3) Bouveault und Blanc, Bl. [3] 3I, 1208 (1904).

4) Vorausgesetzt ist dabei natïrlich, dass bei der Abietinsäure diese Gesetzmässigkeit noch Geltung besitzt.

5) Vergl. unsere Bemerkungen Helv. 4, 506 (1921); 5, 351 (1922). 
möglich an Hand von aromatischen Grundkörpern zu ermitteln. Die Bestimmung der Lage der neuen Methylgruppe im Methylreten wird so die Kenntnis der Stellung des Carboxyls im Abietinsäureskelett vermitteln. Wir werden darüber eingehender in einer späteren Mitteilung berichten. Die bisherigen Versuche haben diese Frage schon in einer gewissen Beziehung geklärt. Die Oxydation des Methylretens (VI) mit Chromsäure liefert nämlich ein Methylreten-chinon (VII),

$\mathrm{CH}_{3} \cdot \mathrm{C}_{16} \mathrm{H}_{15}\left\{\begin{array}{l}-\mathrm{CH} \\ \mathrm{H}\end{array} \longrightarrow \mathrm{CH}_{3} \cdot \mathrm{C}_{16} \mathrm{H}_{15}\left\{\begin{array}{l}-\mathrm{CO} \\ -\mathrm{CO}\end{array} \longrightarrow \mathrm{CH}_{3} \cdot \mathrm{C}_{16} \mathrm{H}_{15}\left\{\begin{array}{l}-\mathrm{C}=\mathrm{N} \\ -\mathrm{C}=\mathrm{N}\end{array} \mathrm{C}_{6} \mathrm{H}_{4}\right.\right.\right.$

VI.

VII.

VIII.

das mit o-Phenylendiamin ins Methylreten-chinoxalin (VIII) übergeht, also ein o-Chinon sein muss. Daraus folgt klar, dass der neuen Methylgruppe im Methylreten und somit auch dem Carboxyl der Abietinsäure eine der Stellungen 2, 3, 4, 5, 6 oder 8 im Retenring (IX) za-

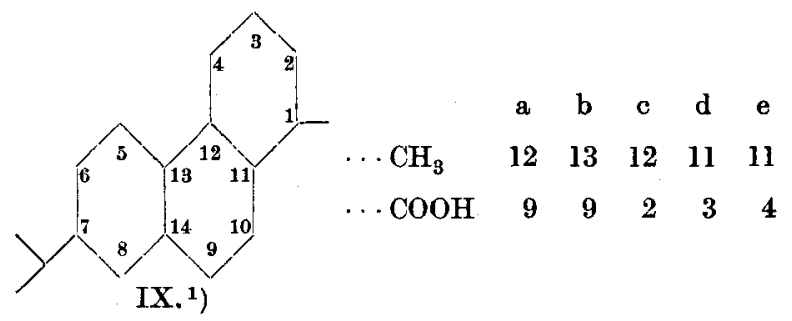

kommen muss. Das Methylretenchinon wäre danach als 1, $x$-Dimethyl7-isopropyl-phenanthrenchinon- $(9,10)$ zu bezeichnen.

Geht man von der Annahme aus ${ }^{2}$ ), dass die Abietinsäure als Diterpenabkömmling analog wie alle Mono- und Sesquiterpenverbindungen mit bekanntem Skelett ${ }^{3}$ ) aus Isoprenresten zusammengesetzt ist, und dass die bei der Dehydrierung der Abietinsäure abgespaltene Methylgruppe $\left(\ldots \mathrm{CH}_{3}\right)$ tertiär und das Carboxyl (..COOH) sekundär gebunden sein muss, so wären für das Abietinsäureskelett nur fünf $(a-e)$ verschiedene Anordnungen möglich, die in der obigen Tabelle zusammen-

1) Für die Numerierung des hydrierten Phenanthrenringes machen wir obigen Vorschlag unter Beibehaltung der Phenanthren-numerierung, um so Missverständnisse zu vermeiden.

2) Helv. 5, 317 (1922).

3) Vergl. Helv. 5, 354 (1922). Auch Verbindungen wie Camphen, Fenchon, aSantalen, die keinen p-Cymolring aufweisen, lassen sich schematiech in Isoprenreste zerlegen. 
gestellt sind. Da durch die Konstitution des Methylreten-chinons die Schemata $a$ und $b$ ausgeschlossen werden, bliebe noch zwischen den letzten drei $(c-e)$ eine Entscheidung zu treffen.

Es sei noch betont, dass wir dazu übergegangen sind, die in dieser Arbeit eingeschlagene neue Methodik auch für die Konstitutionsaufklärung anderer polycyclischer, in der Natur vorkommenden Verbindungen and ihrer Abbauprodukte in geeigneten Fällen anzuwenden. Insbesondere dürfte die Übertragung unserer Reaktionen auf die anderen Harzsäuren $\mathrm{C}_{20} \mathrm{H}_{30} \mathrm{O}_{2}$ zur Klärung der Isomerieverhältnisse in dieser Reihe beitragen. Auf die Einzelheiten soll in späteren Arbeiten eingegangen werden.

\section{Experimenteller Teil.}

Dehydrierung der Abietinsäure vom Smp. $158^{\circ}$ mit Schwefel $\left.{ }^{1}\right)$.

(Gleichung I.)

$48 \mathrm{gr}$ Abietinsäure $^{2}$ ) wurden mit 24 gr Schwefel verrieben und im Ölbade allmählich von $180-250^{\circ}$ erhitzt, bis nach einigen Stunden die Gasentwicklung aufhört. Das Reaktionsprodukt wurde direkt im Vakuum destilliert, wobei das mit den ersten Anteilen übergehende Reten rasch erstarrte. Der Nachlauf besteht aus einer zähen, nicht krystallisierenden Masse. Zur Aufarbeitung wurde das ganze Destillat in Äther aufgenommen und mit verdünnter Natronlauge ausgeschüttelt. Beim Ansäuern der alkalischen Lösung fällt eine zähe, amorphe, stark schwefelhaltige Masse aus.

Der neutrale Anteil wurde mehrmals aus Alkohol umkrystallisiert und lieferte so das in farblosen Blättchen krystallisierende reine Reten vom Smp. 98 . Die in der alkoholischen Mutterlauge enthaltenen, teilweise verschmierten Krystalle können durch Destillation im Vakuum über Natrium gereinigt werden und geben so ein fast völlig erstarrendes Destillat von Reten, das nach einmaligem Umkrystallisieren den richtigen Smp. aufweist. Mit alkoholischer Pikrinsäure wurde daraus das bekannte, bei $124^{\circ}$ schmelzende Pikrat erhalten.

Zur weiteren Charakterisierung des Retens wurde das noch unbekannte Styphnat hergestellt durch Mischen der konzentrierten

\footnotetext{
1) In der Hauptsache entsprechend den Beobachtungen von Vesterberg, B. 36, 4200 (1903).

2) Dargestellt nach Helv. 5, 328 (1922).
} 
heissen Lösungen von Reten und Trinitroresorcin in Alkohol. Das aus einem gelben Krystallpulver bestehende Styphnat schmilzt nach dem Umkrystallisieren aus Alkohol bei $142^{\circ}$.

0,1298 gr Subst. gaben $0,2870 \mathrm{gr} \mathrm{CO}_{2}$ und $0,0546 \mathrm{gr} \mathrm{H}_{2} \mathrm{O}$

$\mathrm{C}_{24} \mathrm{H}_{21} \mathrm{O}_{8} \mathrm{~N}_{3}$ Ber. C 60,13 H $4,38 \%$

Gef. , 60,32 , 4,70\%

Dehydrierung des Abietinsäure-methylesters ${ }^{1}$ ) mit Schwefel.

(Gleichung III.)

14,8 gr des Esters wurden mit 7,1 gr Schwefel allmählich von 180-250 erhitzt, wobei ausser gasförmigen Produkten auch eine geringe Menge einer nicht näher untersuchten, leicht flüchtigen Flüssigkeit entweicht. Beim Destillieren $(15 \mathrm{~mm}$ ) erstarren die ersten Anteile, während der Nachlauf ölig bleibt. Die ganze Masse wurde in Äther gelöst und mit verdünnter Natronlauge ausgezogen. Beim Ansäuern der alkalischen Lösung wurde 0,5 gr eines öligen Produktes gewonnen, das nicht näher untersucht wurde.

Der 5,9 gr betragende neutrale Anteil wurde nochmals bei $12 \mathrm{~mm}$ destilliert, wobei 4 gr eines fast völlig krystallisierenden Produktes bei $228-236^{\circ}$ übergingen. Ein kleiner, bis $255^{\circ}$ siedender Nachlauf blieb ölig. Die Hauptfraktion liefert beim Umkrystallisieren aus Alkohol reines Reten vom Smp. und Mischprobe 98.

\section{Dehydrierung der Dihydro-abietinsäure mit Schwefel.}

(Gleichung II.)

34,1 gr der Dihydro-abietinsäure vom Smp. ca. $150^{\circ 2}$ ) wurden mit 21,5 gr Schwefel in der oben bei der Dehydrierung der Abietinsäure beschriebenen Weise behandelt. Bei der Aufarbeitung wurden analoge Beobachtungen gemacht. Man erhält so 7,0 gr eines neutralen Anteils, der teilweise erstarrt. Nach mehrmaligem Umkrystallisieren aus Alkohol wird ein bei $94^{\circ}$ schmelzender Kohlenwasserstoff erhalten, der mit Reten gemischt den Smp. $96^{\circ}$ zeigt. Das Pikrat desselben schmilzt bei $122-124^{\circ}$ und das Styphnat bei 138-139 ${ }^{\circ}$. Mit den entsprechenden Derivaten des Retens aus der Abietinsäure werden bei der Mischprobe Schmelzpunktserhöhungen beobachtet.

1) Hergestellt mit Dimethylsulfat, vergl. Helv. 5, 330 (1922).

2) Hergestellt durch katalytische Reduktion in alkoholischer Lösung, vergl. Helv. 5, 331 (1922). 
 \\ Darstellung des Abietinols.

$$
\mathrm{C}_{20} \mathrm{H}_{32} \mathrm{O}
$$

110 gr Abietinsäure-methylester wurden in $130 \mathrm{~cm}^{3}$ abs. Alkohol gelöst und zu 165 gr Natrium in dünnem Strahle durch einen Tropftrichter zulaufen gelassen. Zu dem im Ölbade auf $120^{\circ}$ erhitzten Gemisch wurde unter Rühren mit der Turbine im Laufe von fünf Stunden 11/2 Liter Alkohol zugetropft. Nachdem das Natrium aufgelöst war wurden $300 \mathrm{~cm}^{3}$ Wasser eingetragen und zur Verseifung des nicht reduzierten Anteils des Esters noch zwei Stunden gekocht. Zur Aufarbeitung wurde der Äthylalkohol mit Wasserdampf abgeblasen, der Rückstand mit Wasser versetzt und das abgeschiedene Öl in Äther aufgenommen. Um das Natriumsalz der Abietinsäure völlig zu entfernen, wird die ätherische Lösung mehrmals mitWasser durchgeschüttelt. Aus den gesammelten wässrig alkalischen Auszügen kann durch Ansäuern der unveränderte Anteil der angewandten Abietinsäure regeneriert werden. Diese Säure schmolz nach zweimaligem Umkrystallisieren aus Aceton bei $155-157^{\circ},[\alpha]_{\mathrm{D}}=-61^{\circ}$ (in 5-proz. alkohol. Lösung).

$0,1177 \mathrm{gr}$ Subst. gaben $0,3417 \mathrm{gr} \mathrm{CO}_{2}$ und 0,1046 gr $\mathrm{H}_{2} \mathrm{O}$

$\mathrm{C}_{20} \mathrm{H}_{30} \mathrm{O}_{2}$ Ber. C 79,41 H $10,01 \%$

Gef. , 79,20 , $9,94 \%$

Das in Äther gelöste neutrale Produkt liefert bei der Destillation $68 \mathrm{gr}$ einer einheitlich bei $178-182^{\circ}(3 / 4 \mathrm{~mm})$ siedenden farblosen zähen Masse, die aus reinem Abietinol besteht (68\% Ausbeute). Der Destillationsrückstand ist nur ganz unbedeutend. Zur Analyse wurde nochmals destilliert und dabei ein Sdp. 169-172 $(0,2 \mathrm{~mm})$ beobachtet. In Schwefelkohlenstofflösung wird Brom vom Abietinol momentan entfärbt. Mit der molekularen Menge Phenylisocyanat tritt auch bei langem Stehen keine Krystallbildung ein.

$$
\mathrm{d}_{4}^{17}=1,0305 \quad \mathrm{n}_{\mathrm{D}}^{17}=1,5487 \quad \mathrm{M}_{\mathrm{D}}=88,95
$$

$\mathrm{M}_{\mathrm{D}}$ ber. für $\mathrm{C}_{20} \mathrm{H}_{3 \varepsilon} \mathrm{O} / \overline{\overline{2}}=88,55$, woraus folgt $\mathrm{EM}_{\mathrm{D}}=0,40$

0,1016 gr Subst. gaben $0,3090 \mathrm{gr} \mathrm{CO}_{2}$ und $0,1022 \mathrm{gr}_{2} \mathrm{O}$

$0,0849 \mathrm{gr}$ Subst. gaben $0,2582 \mathrm{gr}$, und $0,0836 \mathrm{gr} \mathrm{H}_{2} \mathrm{O}$

$$
\begin{array}{lll}
\mathrm{C}_{20} \mathrm{H}_{32} \mathrm{O} & \begin{array}{l}
\text { Ber. C } 83,26 \\
\text { Gef. , } 83,01 ; 82,96
\end{array} & \text { H } 11,19 \% \\
& \text {, } 11,26 ; 11,02 \%
\end{array}
$$

Beim Kochen mit 20-proz. alkohol. Schwefelsäure spaltet Abietinol kein Wasser ab. 
Reaktionen des Abietinols.

Dehydrierung. 15,7 gr Abietinol wurden mit $7 \mathrm{gr}$ Schwefel in der beschriebenen Weise dehydriert, wobei auch eine Wasserabspaltung vor sich geht. Bei der nachherigen Destillation werden 5 gr eines gelben dickflüssigen Öls erhalten, das bald grösstenteils krystallisierte. Die nach dem Digerieren mit Alkohol abfiltrierten Krystalle schmolzen nach dem Umkrystallisieren aus Alkohol bei $98-99^{\circ}$ und erweisen sich nach der Mischprobe als identisch mit Reten.

Dihydro-abietinol. 7,0 gr Abietinol wurden nach FokinWillstätter in alkoholischer Lösung in Gegenwart von Platinschwarz mit Wasserstoff behandelt. Das Redaktionsprodukt siedet einheitlich bei $169-171^{\circ}(0,5 \mathrm{~mm})$ als farblose zähe Masse, von der eine Anfangs(a) und eine Endfraktion (b) analysiert wurde.

a) 0,1051 $\mathrm{gr}$ Subst. gaben $0,3188 \mathrm{gr} \mathrm{CO}_{2}$ und $0,1117 \mathrm{gr}_{2} \mathrm{O}$

b) $0,0946 \mathrm{gr}$ Subst. gaben $0,2862 \mathrm{gr}$, und $0,0985 \mathrm{gr}$,

$$
\begin{array}{lll}
\mathrm{C}_{20} \mathrm{H}_{34} \mathrm{O} & \text { Ber. C 82,68 } & \text { H } 11,81 \% \\
& \text { Gef. ,, 82,75; 82,54 } & \text {, } 11,89 ; 11,65 \%
\end{array}
$$

Überführung des Abietinols ins Methyl-abietin $\mathrm{C}_{20} \mathrm{H}_{30}$.

\section{(Gleichung IV.)}

Versuch a. 65 gr Abietinol wurden in $500 \mathrm{~cm}^{3}$ Petroläther unter Eiskühlung allmählich mit der gleichen Gewichtsmenge Phosphorpentachlorid versetzt, wobei heftige Chlorwasserstoffentwicklung stattfindet. Nach mehrstündigem Stehen wurde noch eine Stunde am Wasserbade gekocht. Zur Aufarbeitung wurde die schwach gefärbte Petrolätherlösung auf Eis gegossen und mehrmals abwechselnd mit Wasser und verdünnter Natronlauge ausgeschüttelt. Es werden dabei saure phosphorhaltige Nebenprodukte in Form seifiger Lösungen entfernt.

Das neutrale Reaktionsprodukt wird im Hochvakuum destilliert. Nachdem eine anfangs stattfindende heftige Gasentwicklung aufgehört hat, siedet die Hauptmenge bei etwa $140-150^{\circ}(0,5 \mathrm{~mm})$. Daneben wird noch ein bis $170^{\circ}$ siedender Nachlauf erhalten. Durch nochmaliges Fraktionieren werden $27 \mathrm{gr}$ des bis $150^{\circ}(0,5 \mathrm{~mm})$ siedenden Anteils und $10 \mathrm{gr}$ Nachlauf erhalten. Die unscharf siedende chlorhaltige höhere Fraktion wurde vorläufig nicht näher untersucht. 
Nachdem eine quantitative Halogenbestimmung ergab, dass die tiefere Fraktion nur unwesentliche Mengen Chlor enthält, wurde sie zur Reinigung über Natrium destilliert, wobei 25 gr bei $147-152^{\circ}$ $(0,8 \mathrm{~mm})$ als dickflüssiges farbloses chlorfreies öl übergingen. Es konnte daraus kein Pikrat erhalten werden. Mit Kaliumpermanganat in Acetonlösung tritt leicht Oxydation ein; ebenso wird auch Brom in Schwefelkohlenstoff rasch entfärbt.

$$
\begin{gathered}
\alpha_{D}=+56,2^{0} \quad d_{4}^{17}=0,9750 \quad \mathrm{n}_{\mathrm{D}}^{17}=1,54435 \quad \mathrm{M}_{\mathrm{D}}=87,48 \\
\mathrm{M}_{\mathrm{D}} \text { ber. für } \mathrm{C}_{20} \mathrm{H}_{30} \sqrt{3}=86,55 \quad \mathrm{EM}_{\mathrm{D}}=0,93 \\
0,1008 \text { gr Subst. gaben } 0,3283 \text { gr } \mathrm{CO}_{2} \text { und } 0,0983 \mathrm{gr} \mathrm{H}_{2} \mathrm{O} \\
\mathrm{C}_{20} \mathrm{H}_{30} \quad \text { Ber. C } 88,82 \quad \mathrm{H} 11,18 \% \\
\text { Gef. , } 88,90 \quad, 10,91 \%
\end{gathered}
$$

Nach dem Kochen des Methyl-abietins mit 20-proz. alkoholischer Schwefelsäure zeigt das regenerierte Produkt das gleiche Verhalten gegen Kaliumpermanganat und Brom wie vorher.

Versuch b. Der aus 118 gr Abietinol nach dem unter a) bebeschriebenen Verfahren erhaltene neutrale Anteil des Chlorierungsproduktes wurde undestilliert mit der 1 1/2-fachen Menge Chinolin eine halbe Stunde auf $250-260^{\circ}$ erhitzt. Aufgearbeitet wurde durch Aufnehmen in Äther und mehrmaliges Ausschütteln mit verdünnter Salzsäure. Da sich bei der Destillation des Produkts auch der Nachlauf als chlorfrei erwies, wurde das ganze Destillat zur Reinigung über Natrium destilliert, wobei die Hauptmenge (52 gr) bei $145-147^{\circ}$ $(0,5 \mathrm{~mm})$ überging. Die Eigenschaften sind die gleichen wie die im Versuch a.

$$
\begin{aligned}
& \mathrm{d}_{4}^{15}=0,9795 \quad \mathrm{n}_{\mathrm{D}}^{15}=1,5477 \quad \mathrm{M}_{\mathrm{D}}=87,52 \quad \mathrm{M}_{\mathrm{D}} \text { ber. für } \mathrm{C}_{20} \mathrm{H}_{30} \sqrt{3}=86,55 \\
& \mathrm{EM}_{\mathrm{D}}=0,97
\end{aligned}
$$

Dehydrierung des Methyl-abietins zum Methylreten $C_{19} H_{20}$. (Gleichung V.)

63,5 gr Methyl-abietin wurden mit 30 gx Schwefel (entsprechend 4 Atomen) vier Stunden auf $190-200^{\circ}$ erhitzt, wobei ruhige Gas- 
entwicklung stattfindet. Schliesslich wird die Temperatur im Laufe von weiteren zwei Stunden bis auf $270^{\circ}$ gesteigert. Nach Beendigung der Gasentwicklung wird das Reaktionsprodukt bei $15 \mathrm{~mm}$ destilliert, wobei $26 \mathrm{gr}$ eines dickflüssigen bräunlichen Öls von $210-240^{\circ}$ übergehen. Das schwefelhaltige Destillat wurde in Äther aufgenommen und mit verdünnter Kalilauge mehrmals ausgeschüttelt; beim Ansäuern der alkalischen Lösung tritt nur eine schwache Trübung ein. Der neutrale Anteil wurde nochmals bei $15 \mathrm{~mm}$ in drei Fraktionen zerlegt:

$$
\begin{aligned}
& \text { 1. Sdp. } 210-220^{\circ}, 10 \mathrm{gr} \\
& \text { 2. ", } 220-230^{\circ}, 10,3 \mathrm{gr} \\
& \text { 3. , } 230-235^{\circ}, 4,5 \mathrm{gr}
\end{aligned}
$$

Alle drei Fraktionen sind schwach bräunlich gefärbt und zeigen einen unangenehmen Geruch. Die Fraktion' 3 ist zähflüssiger als die ersten Anteile. Nach kurzem Stehen krystallisiert die Fraktion 2 zu einem dicken Brei und nach dem Impfen mit demselben scheiden sich auch aus den beiden anderen Fraktionen Krystalle ab. Auch beim Auflösen der Fraktionen 1 und 2 in heissem absoluten Alkohol und Erkalten tritt Krystallisation ein. Das so erhaltene Methylreten schmilzt bei ca. $75^{\circ}$. Um aus den öligen Anteilen das Methylreten möglichst vollständig abzutrennen, wurden dieselben mit einer heissen alkoholischen Lösung von $25 \mathrm{gr}$ Pikrinsäure versetzt und in einer Schale verdunsten gelassen. Der mit Öl durchtränkte Krystallbrei wurde auf Ton gepresst und durch Erwärmen mit Ammoniak am Wasserbade zerlegt. Nach dem Aufnehmen in Äther, Schütteln mit Natronlauge und Wasser, sowie nachheriges Destillieren wird wieder ein teilweise krystallisierendes Produkt erhalten. Die Krystalle sind mit obigen identisch. Nach einmaligem Umkrystallisieren aus Alkohol steigt der Smp. auf $79^{\circ}$ und blieb auch nach weiterem zweimaligem Umlösen konstant. Die Ausbeute beträgt etwa $10 \%$ der Theorie. Das Methyl-reten besteht wie auch das Reten aus farblosen glänzenden Blättchen.

0,08715 gr Subst. gaben $0,2932 \mathrm{gr}_{2} \mathrm{CO}_{2}$ und $0,0638 \mathrm{gr} \mathrm{H}_{2} \mathrm{O}$ 0,0986 gr Subst. gaben $0,3328 \mathrm{gr}$,, und $0,0714 \mathrm{gr}_{2} \mathrm{O}$

$$
\begin{array}{lll}
\mathrm{C}_{19} \mathrm{H}_{20} & \text { Ber. C 91,86 } & \mathrm{H} 8,14 \% \\
& \text { Gef. ., 91,81; 92,06 } & , 8,19 ; 8,10 \%
\end{array}
$$




\section{Derivate des Methylretens (Formel VI.)}

Pikrat. Beim Zusammengeben molekularer Mengen von Methylreten und Pikrinsäure in heisser konzentrierter alkoholischer Lösung entsteht nach dem Erkalten eine dunkelorange gefärbte Fällung, die wohl das Pikrat des Methylretens darstellt. Dasselbe ist aber wenig beständig und zerfällt schon beim Umkrystallisieren und Waschen mit Alkohol teilweise in die Komponenten, indem das abgeschiedene Pikrat mit Krystallen des Methylretens durchsetzt erscheint. Es wurde daher auf die Analyse verzichtet. Versetzt man Methylreten mit überschüssiger Pikrinsäure, so entstehen Fällungen, die auf $1 \mathrm{Mol}$. des Kohlenwasserstoffs 2-3 Mol. Pikrinsäure enthalten, sich anscheinend ohne Zersetzung in die Komponenten aus Alkohol umkrystallisieren lassen und beim Erhitzen äusserst heftig explodieren.

Methylreten-chinon (Formel VII). 3,1 gr Methylreten wurden in $9 \mathrm{~cm}^{3}$ Eisessig durch Erwärmen am Wasserbade gelöst und bei ca. 50-60 allmählich eine Lösung von $6,2 \mathrm{gr}$ Chromtrioxyd in $35 \mathrm{~cm}^{3}$ Eisessig zugegeben. Das Reaktionsgemisch wird dann noch drei Stunden am kochenden Wasserbade erhitzt und der nach dem Erkalten nach eintägigem Stehen abgeschiedene Krystallbrei filtriert und mit eiskaltem 80-proz. Alkohol einigemal ausgewaschen. Die so erhaltenen $1,3 \mathrm{gr}$ Methylreten-chinon zeigen den Smp. von ca. $143^{\circ}$. Nach zweimaligem Umkrystallisieren aus Alkohol wird dasselbe in Form roter Blättchen vom konstant bleibenden Smp. $147^{\circ}$ erhalten. Es ist analog dem Retenchinon ${ }^{1}$ ) sehr schwer verbrennlich; bei der Analyse können nur richtige Kohlenstoffwerte erhalten werden, wenn die Substanz mit gepulvertem Bleichromat verrieben und stark geglüht wird.

0,0919 gr Subst. gaben $0,0522 \mathrm{gr}_{2} \mathrm{O}$

0,0615 gr Subst. gaben $0,1848 \mathrm{gr} \mathrm{CO}_{2}$ und $0,0401 \mathrm{gr} \mathrm{H}_{2} \mathrm{O}$

$$
\begin{array}{lll}
\mathrm{C}_{19} \mathrm{H}_{18} \mathrm{O}_{2} & \text { Ber. C 81,98 } & \text { H 6,53\% } \\
& \text { Gef. , 81,98 } & \text {, 6,36; 7,29\% }
\end{array}
$$

Methylreten-chinoxalin (Formel VIII). Eine konzentrierte Lösung von $0,46 \mathrm{gr}$ Methylreten-chinon in Eisessig wird unter Erwärmen am Wasserbade mit einer alkoholischen Lösung von 0,2 gr o-Phenylendiamin versetzt. Nach kurzem Erwärmen beginnt die Abscheidung des Methylreten-chinoxalins und nach dem Erkalten ist die ganze Lösung von den verfilzten Nädelchen durchsetzt. Das Roh-

1) Vergl. Bamberger und Hooker, A. 229, 118 (1885). 


\title{
$-593-$
}

produkt schmilzt bei $163^{\circ}$ und nach dem Umkrystallisieren aus einem Gemisch von Chloroform und absolutem Alkohol wird der konstant bleibende Smp. von $165^{\circ}$ erreicht. Die fast farblosen Nädelchen ergeben mit dem ähnlich aussehenden und schmelzenden Reten-chinoxalin gemischt eine Schmelzpunktsdepression von ca. $10^{\circ}$.

$$
\begin{aligned}
& \left.0,0884 \mathrm{gr} \text { Subst. gaben }{ }^{1}\right) 0,2765 \mathrm{gr} \mathrm{CO}_{2} \text { und } 0,0549 \mathrm{gr} \mathrm{H}_{2} \mathrm{O} \\
& \mathrm{C}_{25} \mathrm{H}_{22} \mathrm{~N}_{2} \text { Ber. C 85,67 H 6,33\% } \\
& \text { Gef. , } 85,34 \quad, 6,95 \%
\end{aligned}
$$

Zürich, Chem. Institut der Eidg. Techn. Hochschule.

\section{Ueber den Einfluss von Weehselstromüberlagerung auf das Abscheidungs- und Auflösungspotential des Nickels.}

\author{
von \\ V. Kohlsehatter und H. Schődl.
}

(29. V. 22.)

Die folgenden Potentialmessungen wurden im Anschluss an die Untersuchungen über die Struktur von Nickelniederschlägen ausgeführt, über die in der vorausgehenden Abhandlung ${ }^{2}$ ) berichtet wurde, haben aber auch ausserhalb dieses Zusammenhanges für die Kenntnis des elektrolytischen Verhaltens des Nickels einiges Interesse. Allerdings sind sie durch ihre Veranlassung insofern spezialisiert, als sie sich auf die Bedingungen beschränken, unter denen die strukturell untersuchten Niederschläge gewonnen wurden; insbesondere sind sie nicht bei Luft-

1) Mit Bleichromat gemischt.

2) Helv. 5, 490 (1922). 